\title{
A Matched Case-control Study of the Association between Low-dose Aspirin and Peptic Ulcers in Patients without a History of Peptic Ulcers
}

\author{
Atsushi Tanabe, Makoto Sasaki*, Yoshitsugi Ito, Akihiro Shimozato, Kazunori Adachi, Noriko Okaniwa, Shigeki, Goji, \\ Hisastsugu Noda, Yoshiharu Yamaguchi, Yoshihiro Kondo,Shinya Izawa, Masahide Ebi, Naotaka Ogasawara, Yasushi \\ Funaki, Kunio Kasugai \\ Aichi Medical University School of Medicine
}

Received: August 08, 2015; Accepted: September 10, 2015; Published: October 28, 2015

*Corresponding author: Makoto Sasaki, Department of Gastroenterology, Aichi Medical University School of Medicine, Tel: +81-561623311, Fax: +81561621508, E-mail: msasaki@aichi-med-u.ac.jp

\begin{abstract}
Background: Long-term administration of low-dose aspirin (LDA) is associated with greater risk of adverse events, including gastro duodenal ulcers. This study aimed to assess the risk factors and efficacy of medications to prevent the development of peptic ulcer disease in Japanese patients without prior history of peptic ulceration.

Methods: We conducted a matched case-control study using esophagogastroduodenoscopy records collected from January through December 2010. We enrolled 219 consecutive outpatients receiving LDA (100 mg) and 219 age- and sex-matched controls who did not receive LDA and did not have a prior history of peptic ulcer. Clinical parameters, reason for endoscopy, and endoscopic findings were analyzed.
\end{abstract}

Results: A significantly higher number of patients receiving LDA compared to those not receiving LDA were diagnosed endoscopically with peptic ulceration $(20$ [9.1\%] vs. 7 [3.2\%]; odds ratio [OR], 3.0; 95\% confidence interval [CI], 1.26-7.35; $\mathrm{p}=0.016$ ). Multiple logistic regression analysis identified male sex, abdominal symptoms, and non-steroidal anti-inflammatory drug (NSAID) and LDA use and PPI use as risk and protective factors, respectively, for peptic ulcer (OR, $4.7,95 \% \mathrm{CI}, 1.28-17.64, \mathrm{p}=0.020 ; \mathrm{OR}, 2.5,95 \% \mathrm{CI}, 1.01-6.12, \mathrm{p}=0.047$ OR, 6.8, 95\% CI, 1.72-26.40, p=0.006; OR, 9.72; 95\% CI, 2.64-35.72, $\mathrm{p}=0.006$; and $\mathrm{OR}, 0.1,95 \% \mathrm{CI}, 0.04-0.57, \mathrm{p}=0.006$, respectively).

Conclusion: LDA and NSAID use increases the risk of peptic ulcer even in patients without peptic ulcer history; proton pump inhibitors reduce the risk of developing gastric or duodenal ulcers. These results may help identify patients who require interventions for the prevention of NSAID- or LDA-induced peptic ulcers.

Keywords: aspirin, peptic ulcer, prevention, proton pump inhibitors, risk factors

\section{Introduction}

The use of low-dose aspirin (LDA; 75-325 mg daily) contributes to the prevention of thrombi and emboli in patients with ischemic heart disease or ischemic cerebrovascular disease [1]. However, LDA has been shown to cause gastrointestinal damage
[2] and be associated with increased risk of gastroduodenal ulcers and their potentially fatal complications (e.g., gastrointestinal bleeding and perforation) [3]. In case-control studies, the odd ratios (ORs) of bleeding ranges 2.4-7.7 [4, 5], which is similar to that seen in regular users of non-steroidal anti-inflammatory drugs (NSAIDs) [6]. The mortality attributable to the use of NSAIDs or LDA is approximately 20-25 cases per million people, and one-third of these cases are attributable to LDA [7].

The identified risk factors for ulcer bleeding owing to aspirin use are history of ulcer bleeding; aspirin dose; advanced age ( $>70$ years); concomitant use of NSAIDs or anticoagulants; use of dual antiplatelet therapy; Helicobacter pylori (H. pylori) infection; and history of alcohol abuse, diabetes, or renal failure [6, 8]. Proton pump inhibitors (PPIs) are used to decrease LDA-associated gastro duodenal mucosal and NSAID-induced injuries [9-11]. In Japan, since 2011 and 2013, treatment with half-dose PPI (lansoprazole $15 \mathrm{mg} /$ day) and full-dose PPI (esomeprazole $20 \mathrm{mg}$ /day) for the prevention of NSAID- or LDA-induced peptic ulcers, respectively, has been permitted as a medical service under health insurance for patients with a history of peptic ulcers that is associated with a high risk for the future development of peptic ulcers. Yeomans et al. [12] stated that the use of esomeprazole $20 \mathrm{mg}$ reduces the risk of developing LDA-associated gastric and/or duodenal ulcers in elderly patients without preexisting gastro duodenal ulcers. However, only a few studies have reported a reduced risk of LDA-associated peptic ulcers owing to the use of PPIs in patients without preexisting peptic ulcers. Therefore, the aim of this retrospective case-control study was to clarify the risk and effect of PPIs on peptic ulcer development in Japanese patients receiving LDA who did not have preexisting peptic ulcers.

\section{Methods}

This retrospective observation case-control study was conducted at our hospital using esophagogastroduodenoscopy records collected between January and December 2010. This study was approved by the ethics committee of our University. 
Consecutive outpatients taking LDA (100 mg) for $>3$ months (case) and age and sex-matched outpatients not taking LDA (control) were enrolled. All endoscopic examinations were recorded digitally. The endoscopic findings were blindly evaluated by one experienced endoscopist. We defined an ulcer as a mucosal deficit of $>5 \mathrm{~mm}$ in diameter. The exclusion criterion was a history of gastrectomy.

We recorded the following data from patient medical records: clinical characteristics, including sex, age, and smoking and drinking habits; underlying disease (such as hypertension, hyperlipidemia, ischemic heart disease, diabetes mellitus, and cerebrovascular disease); peptic ulcer history; use of concomitant gastric agents; use of NSAIDs; reason for endoscopy (abdominal symptoms [epigastric pain, heartburn, dysphagia, anorexia, nausea] or bleeding signs [anemia, hematemesis, tarry stool]); and endoscopic findings.

The criterion of no preexisting gastroduodenal ulcers was defined as no peptic ulcer history on the basis of medical records and no evidence of peptic ulcer scarring on endoscopy. Gastric mucosal atrophy was scored, using endoscopy, on a 6-grade scale (C1, C2, C3, 01, 02, and 03; C, closed; 0, open) according to Kimura and Takemoto's classification [13]. The presence of gastric mucosal atrophy was defined as an endoscopic score of C3-03.

The patients' demographic and characteristics were compared between the case and control groups, using the Mantel-Haenszel test. The ORs and 95\% confidence interval (CI) were obtained by Mantel-Haenszel statistics and multiple logistic regression analysis to identify risk or preventive factors. A two-sided $\mathrm{P}$ value of less than 0.05 was considered statistically significant. All statistical computations were performed using Microsoft Excel 2013® software (Microsoft Corp., Redmond, WA, USA).

\section{Results}

A total of 438 patients (219 cases and 219 controls) were enrolled in this study. Patient demographic and clinical characteristics are shown in Table 1. Age, sex, current smoking status, endoscopic gastric mucosal atrophy, and reason for endoscopy were not significantly different between case and control groups. As the underlying disease, hypertension, hyperlipidemia, ischemic heart disease, and diabetes mellitus was more frequent in the case group than the control group. The frequency of abdominal symptoms, as a reason for endoscopy, was not significantly different between the case and control groups. However, anemia were more frequent in the case group than in the control group (Table 1).

Out of 219 case patients and 219 control patients, 20 (9.1\%) and 7 (3.2\%) patients, respectively, were endoscopically diagnosed with peptic ulcers. The risk of peptic ulcer in the case group was 3 times higher than in the control group (Table 2). On multivariate analysis, male sex, abdominal symptoms, NSAIDs, and LDA were identified as risk factors for peptic ulcers (odds ratio [OR]: 4.74; 95\% CI: 1.28-17.64; p=0.020, OR: 2.49; 95\% CI:
1.01-6.12: $\mathrm{p}=0.047$, OR: $6.75 ; 95 \%$ CI: $1.72-26.40 ; \mathrm{p}=0.006$, and OR: 9.72; 95\% CI: 2.64-35.72; $\mathrm{p}=0.001$, respectively). PPI was identified as a protective factor of peptic ulcer (OR: $0.14,95 \% \mathrm{CI}$ : 0.04-0.57, p=0.006) (Table 3).

\section{Discussion}

To our knowledge, there is only one prospective trial investigating the risk of primary peptic ulcer development in patients taking LDA with or without concomitant acid suppressive therapy [12]. In that trial, only patients without preexisting peptic ulcers were enrolled, and 26-week treatment with esomeprazole at $20 \mathrm{mg}$ once daily significantly reduced the risk of peptic ulcer formation in patients taking LDA for the secondary prevention of cardiovascular events. However, the patients were $\geq 60$ years of age and tested negative for H. pylori. In our study, we retrospectively investigated the risk of LDA-induced peptic ulcer in a restricted population of patients without preexisting peptic ulcers in comparison with a matched background population.

In a Japanese epidemiologic study, there was minimal biological interaction between $H$. pylori infection and NSAIDs with respect to bleeding [5], and NSAIDs and $H$. pylori infection seem to be independent risk factors for peptic ulcers and bleeding. In this retrospective study, only 12 patients tested positive for $H$. pylori infection, 16 patients tested negative, and the results in the remaining 410 patients were inconclusive; therefore, we could not investigate the influence of $H$. pylori infection on peptic ulcer development. In the present study, previous eradication therapy was not confirmed in 410 (93.6\%) patients, and 244 (55.7\%) patients were found to have endoscopic gastric mucosal atrophy. This result suggests that the $H$. pylori infection rate was high in the present study. Previous studies have documented a risk of peptic ulcer complications owing to upper gastrointestinal bleeding [8, 14-16]; however, there were only a few reports on patients without preexisting peptic ulcers. Yeomans et al. reported that peptic ulcer prevalence was $7.1 \%$ in patients who received LDA. In the present study, analysis was restricted to patients with no peptic ulcer history; peptic ulcer prevalence was $9.1 \%(20 / 219)$, which is slightly higher than the $7.1 \%$ reported in the study of Yeomans et al. [17], which recruited patients who did not receive gastroprotective drugs. In the present study, the risk of peptic ulcers was 3 times higher in patients treated with LDA than in those who did not receive LDA. Moreover, in our study, there were 7 cases of bleeding ( 6 without PPI, 1 with PPI) in 6 patients with gastric ulcers and 1 patient with a duodenal ulcer among those who received LDA (case group). Of the 132 patients who were not taking PPIs, 6 (4.4\%) had gastroduodenal bleeding, a rate that was higher than that for placebo $(1.0 \%)$ in the study by Yeomans et al. Furthermore, there were 3 bleeding cases (all without PPI) in 2 patients with gastric ulcers and 1 patient with a duodenal ulcer among those who did not receive LDA (control group). Of the 132 patients who were not taking PPIs, 3 $(2.3 \%)$ had gastroduodenal bleeding, a rate that was also higher than that for placebo in the study by Yeomans et al., which was treated with LDA. The difference may be the influence of $H$. pylori infection; however, our data are very limited and a further study 
Table 1: Demographic and clinical characteristics of the patients.

\begin{tabular}{|c|c|c|c|}
\hline & Case & Control & \\
\hline & $(n=219)$ & $(n=219)$ & p value \\
\hline Sex (male/female) & $137 / 82$ & $137 / 82$ & \\
\hline Age & $71.2(9.5)$ & $71.6(9.6)$ & 0.925 \\
\hline Hypertension & $125(57.1 \%)$ & $79(36.1 \%)$ & $<0.001$ \\
\hline Hyperlipidemia & $84(38.4 \%)$ & $22(10.0 \%)$ & $<0.001$ \\
\hline Ischemic heart disease & $99(45.2 \%)$ & $3(1.4 \%)$ & $<0.001$ \\
\hline Diabetes mellitus & $43(19.6 \%)$ & $25(11.4 \%)$ & 0.027 \\
\hline Other disease & $53(24.2 \%)$ & $22(10.0 \%)$ & 0.001 \\
\hline Current smoker & $36(16.4 \%)$ & $37(16.9 \%)$ & 1.000 \\
\hline Current alcohol consumer & $25(11.4 \%)$ & $65(29.7 \%)$ & $<0.001$ \\
\hline \multicolumn{4}{|l|}{ Gastric mucosal atrophytric } \\
\hline$(+) \mathrm{C} 3-03$ & $121(55.3 \%)$ & $123(56.2 \%)$ & \\
\hline$(-) 0-\mathrm{C} 2$ & $100(45.7 \%)$ & $98(44.8 \%)$ & 0.924 \\
\hline \multicolumn{4}{|l|}{ Reason for endoscopy } \\
\hline Screening (no symptom) & $129(58.9 \%)$ & $137(62.6 \%)$ & 0.556 \\
\hline Epigastric pain & $19(8.7 \%)$ & $16(7.2 \%)$ & 0.728 \\
\hline Heart burn & $14(6.4 \%)$ & $25(11.4 \%)$ & 0.096 \\
\hline Indigestion & $3(1.4 \%)$ & $3(1.4 \%)$ & 1.000 \\
\hline Abdominal pain & $4(1.8 \%)$ & $1(0.5 \%)$ & 0.373 \\
\hline Nausea and vomiting & $5(2.3 \%)$ & $5(2.3 \%)$ & 1.000 \\
\hline Anorexia & $8(3.6 \%)$ & $9(4.1 \%)$ & 1.000 \\
\hline Dysphagia & $13(5.9 \%)$ & $8(3.7 \%)$ & 0.374 \\
\hline Anemia & $24(11.0 \%)$ & $7(3.2 \%)$ & 0.003 \\
\hline Hematemesis/Tarry stool & $3(1.4 \%)$ & $0(0 \%)$ & 0.249 \\
\hline NSAIDs & $12(5.5 \%)$ & $22(10.0 \%)$ & 0.110 \\
\hline PPI & $87(39.7 \%)$ & $57(26.0 \%)$ & 0.005 \\
\hline $\mathrm{H}_{2} \mathrm{RA}$ & $39(17.8 \%)$ & $22(10.0 \%)$ & 0.030 \\
\hline Cytoprotective gastric agents & $33(15.1 \%)$ & $14(6.4 \%)$ & 0.012 \\
\hline
\end{tabular}

Notes. NSAID = nonsteroidal antiinflammatory drug. PPI = proton pump inhibitor. $\mathrm{H}_{2} \mathrm{RA}=\mathrm{H}_{2}$-receptor antagonist. The data are represented as number (\%), except for age, which is represented as mean (standard error).

Table 2: Prevalence of gastroduodenal ulcers in patients receiving and not receiving low-dose aspirin.

\begin{tabular}{|c|c|c|c|c|c|c|}
\hline \multicolumn{2}{|c|}{ Case } & \multicolumn{3}{|c|}{ Control } & \multicolumn{3}{|c|}{ OR (95\% CI) } & $\mathrm{p}$ value \\
\hline $\mathrm{n}$ & $(\%)$ & $\mathrm{n}$ & $(\%)$ & 3.0 & $(1.26-7.35)$ & 0.016 \\
\hline 20 & $(9.1)$ & 7 & $(3.2)$ & & \\
\hline
\end{tabular}

is necessary to verify our findings.

Gastrointestinal hemorrhage is associated with increased odds of death and severe dependence after acute ischemic stroke and acute coronary syndromes $[18,19]$. Therefore, regardless of the risk of onset, it is necessary to prevent peptic ulcers, which may lead to gastrointestinal bleeding. In the present study, the risk of peptic ulcers was 3 times higher in patients treated with
LDA than those treated without LDA with no previous history of peptic ulcers. Furthermore, male sex, abdominal symptoms, use of NSAIDs, and use of LDA were identified as risk factors of peptic ulcers. Additionally, PPI was identified as a protective factor. Therefore, patients with ischemic heart disease or ischemic cerebrovascular disease as well as thrombi and emboli should be treated with PPI instead of LDA to prevent peptic ulcers. 
Table 3: Risk factors of gastroduodenal ulcers in patients with no history of peptic ulcers.

\begin{tabular}{|l|l|l|l|}
\hline Factors & OR & $\mathbf{9 5 \%}$ CI & p value \\
\hline Male sex & 4.74 & $1.28-17.64$ & 0.020 \\
\hline Advanced age ( $\geq 70$ years) & 2.82 & $0.99-8.09$ & 0.053 \\
\hline Hypertension & 1.2 & $0.43-3.38$ & 0.730 \\
\hline Hyperlipidemia & 1.24 & $0.43-3.66$ & 0.691 \\
\hline Ischemic heart disease & 0.53 & $0.17-1.65$ & 0.271 \\
\hline Diabetes mellitus & 2.17 & $0.90-6.77$ & 0.079 \\
\hline Other disease & 0.39 & $0.07-2.12$ & 0.274 \\
\hline Current smoker & 3.19 & $0.91-11.21$ & 0.070 \\
\hline current alcohol consumer & 0.93 & $0.27-3.26$ & 0.913 \\
\hline Gastric mucosal atrophy & 0.61 & $0.24-1.53$ & 0.290 \\
\hline Abdominal symptom & 2.49 & $1.01-6.12$ & 0.047 \\
\hline NSAIDs & 6.75 & $1.72-26.40$ & 0.006 \\
\hline LDA & 9.72 & $2.64-35.72$ & 0.001 \\
\hline PPI & 0.14 & $0.04-0.57$ & 0.006 \\
\hline H $_{2}$ RA & 0.29 & $0.07-1.30$ & 0.105 \\
\hline Cytoprotective gastric agents & 0.42 & $0.08-2.18$ & 0.302 \\
\hline
\end{tabular}

Notes. $\mathrm{OR}=$ odds ratio. $\mathrm{CI}=$ confidence interval. $\mathrm{NSAID}=$ nonsteroidal antiinflammatory drug. $\mathrm{PPI}=$ proton pump inhibitor. $\mathrm{H} 2 \mathrm{RA}=\mathrm{H}_{2}-$ receptor antagonist. LDA $=$ low-dose aspirin

The results of the present study indicate that LDA is associated with a risk of developing gastroduodenal ulcers and their potentially fatal complications (e.g., gastrointestinal bleeding), even in patients without preexisting peptic ulcers. Because of its retrospective design, the limitations of the present study include the absence of a diagnosis of $H$. pylori infection and ambiguous clinical information, especially with regard to medication history, including NSAIDs use. In our clinic, information about antiplatelets and anticoagulants was easily accessible because these medications were carefully recorded habitually prior to endoscopic examinations. Because this study included only those patients without a history of peptic ulcers, a large-scale and prospective study will be necessary to clarify the risk and protective factors of LDA-associated gastro duodenal mucosal lesions.

LDA increases the risk of developing primary peptic ulcers in Japanese patients, and PPI therapy reduces the risk of developing gastric or duodenal ulcers in patients without preexisting gastro duodenal ulcers. Further studies are necessary to clarify the indication of treatment with PPIs for preventing NSAID- or LDAinduced peptic ulcers in patients without a history of peptic ulcers.

\section{Acknowledgement}

We would like to thank Editage (www.editage.jp) for English language editing.

\section{References}

1. Chan AT, Manson JE, Feskanich D, Stampfer MJ, Colditz GA, Fuchs CS. Long-term aspirin use and mortality in women. Arch Intern Med. 2007;167(6):562-572.

2. Cryer B, Feldman M. Effects of very low dose daily, long-term aspirin therapy on gastric, duodenal, and rectal prostaglandin levels and on mucosal injury in healthy humans. Gastroenterology. 1999;117(1):1725.

3. Garcia Rodriguez LA, Hernandez-Diaz S, de Abajo FJ. Association between aspirin and upper gastrointestinal complications: Systematic review of epidemiologic studies. Br J Clin Pharmacol. 2001;52(5):563571.

4. Lanas A, Bajador E, Serrano P, Fuentes J, Carreno S, Guardia J, et al. Nitrovasodilators, low-dose aspirin, other nonsteroidal antiinflammatory drugs, and the risk of upper gastrointestinal bleeding. N Engl J Med. 2000;343(12):834-839.

5. Sakamoto C, Sugano K, Ota S, Sakaki N, Takahashi S, Yoshida Y, et al. Case-control study on the association of upper gastrointestinal bleeding and nonsteroidal anti-inflammatory drugs in japan. Eur J Clin Pharmacol. 2006;62(9):765-772.

6. Laine L. Approaches to nonsteroidal anti-inflammatory drug use in the high-risk patient. Gastroenterology. 2001;20(3):594-606.

7. Lanas A, Perez-Aisa MA, Feu F, Ponce J, Saperas E, Santolaria S, et al. A nationwide study of mortality associated with hospital admission due to severe gastrointestinal events and those associated with nonsteroidal antiinflammatory drug use. Am J Gastroenterol. 2005;100(8):1685-1693.

8. Moukarbel GV, Signorovitch JE, Pfeffer MA, McMurray JJ, White HD, Maggioni AP, et al. Gastrointestinal bleeding in high risk survivors of myocardial infarction: The valiant trial. Eur Heart J. 2009;30(18):22262232.

9. Hawkey CJ, Karrasch JA, Szczepanski L, Walker DG, Barkun A, Swannell AJ, et al. Omeprazole compared with misoprostol for ulcers associated with nonsteroidal antiinflammatory drugs. Omeprazole versus misoprostol for nsaid-induced ulcer management (omnium) study group. N Engl J Med. 1998;338(11):727-734.

10. Yeomans ND, Tulassay Z, Juhasz L, Racz I, Howard JM, van Rensburg CJ, et al. A comparison of omeprazole with ranitidine for ulcers associated with nonsteroidal antiinflammatory drugs. Acid suppression trial: Ranitidine versus omeprazole for nsaid-associated ulcer treatment (astronaut) study group. N Engl J Med. 1998;338(11):719-726.

11. Chan FK, Chung SC, Suen BY, Lee YT, Leung WK, Leung VK, et al. Preventingrecurrent upper gastrointestinal bleeding in patients with helicobacter pylori infection who are taking low-dose aspirin or naproxen. N Engl J Med. 2001;344(13):967-973.

12. Yeomans N, Lanas A, Labenz J, van Zanten SV, van Rensburg C, Racz I, et al. Efficacy of esomeprazole (20 mg once daily) for reducing the risk of gastroduodenal ulcers associated with continuous use of low-dose aspirin. Am J Gastroenterol. 2008;103(10):2465-2473. doi: 10.1111/j.1572-0241.2008.01995.x.

13. Kimura T, Takemot T. An endoscopic recognition of the atrophic border and its significance in chronic gastritis. Endoscopy. 1969;1(3):87-97.

14. Lanas A, Fuentes J, Benito R, Serrano P, Bajador E, Sainz R. Helicobacter pylori increases the risk of upper gastrointestinal bleeding in patients taking low-dose aspirin. Aliment Pharmacol Ther. 2002;16(4):779- 
786

15. Chan FK. Review: Low-dose aspirin causes a small increase in gastrointestinal bleeding. ACP J Club. 2007;146(1):13.

16. Malfertheiner P, Chan FK, McColl KE. Peptic ulcer disease. Lancet 2009;374(9699):1449-1461

17. Yeomans ND, Lanas AI, Talley NJ, Thomson AB, Daneshjoo R Eriksson B, et al. Prevalence and incidence of gastroduodenal ulcers during treatment with vascular protective doses of aspirin. Aliment Pharmacol Ther. 2005;22(9):795-801.

18. O’Donnell MJ, Kapral MK, Fang J, Saposnik G, Eikelboom JW,
Oczkowski W, et al. Gastrointestinal bleeding after acute ischemic stroke. Neurology. 2008;71(9):650-655. doi: 10.1212/01. wnl.0000319689.48946.25.

19. Nikolsky E, Stone GW, Kirtane AJ, Dangas GD, Lansky AJ, McLaurin B, et al. Gastrointestinal bleeding in patients with acute coronary syndromes: Incidence, predictors, and clinical implications: Analysis from the acuity (acute catheterization and urgent intervention triage strategy) trial. J Am Coll Cardiol. 2009;54(14):1293-1302. doi: 10.1016/j.jacc.2009.07.019. 